\title{
Tabu Search-Based Interactive Fuzzy Stochastic Multi-Level 0-1 Programming
}

\author{
Masatoshi Sakawa*, Takeshi Matsui \\ Faculty of Engineering, Hiroshima University, Higashi-Hiroshima, 739-8527, Hiroshima, Japan \\ *Corresponding Author: sakawa@hiroshima-u.ac.jp
}

Copyright (C)2013 Horizon Research Publishing All rights reserved.

\begin{abstract}
This paper considers interactive fuzzy programming for multi-level 0-1 programming problems involving random variable coefficients both in objective functions and constraints. Following the concept of fractile criterion optimization together with chance constrained programming, the formulated stochastic multi-level 0-1 programming problems are transformed into deterministic ones. Taking into account vagueness of judgments of the decision makers, interactive fuzzy programming is presented. In the proposed interactive method, after determining the fuzzy goals of the decision makers at all levels, a satisfactory solution is derived efficiently by updating satisfactory levels of the decision makers with considerations of overall satisfactory balance among all levels. For solving the transformed deterministic problems efficiently, tabu search for general 0-1 programming problems is introduced. An illustrative numerical example for a three-level 0-1 programming problem is provided to clarify the proposed method.
\end{abstract}

Keywords Multi-level 0-1 programming, Random variables, Interactive fuzzy programming, Fractile criterion optimization, Tabu search

\section{Introduction}

In the real world, we often encounter situations where there are two or more decision makers (DMs) in an organization with a hierarchical structure, and they make decisions in turn or at the same time so as to optimize their objective functions. Such situations are formulated as multi-level programming problems, and the Stackelberg solution has been usually employed as a solution concept [1].

When the Stackelberg solution is employed, it is assumed that there is no communication between the two DMs, or they do not make any binding agreement even if there exists such communication. However, the above assumption is not always reasonable when we model decision making problems in a decentralized firm as a twolevel programming problem in which top management is a leader and an operation division of the firm is a follower because it is supposed that there exists cooperative relationship between them.

For two-level linear programming problems or multilevel ones such that decisions of decision makers in all levels are sequential and all of the decision makers essentially cooperate with each other, Lai [2] and Shih et al. [3] proposed fuzzy interactive approaches. In their methods, the decision makers identify membership functions of the fuzzy goals for their objective functions, and in particular, the decision maker at the upper level also specifies those of the fuzzy goals for the decision variables. The decision maker at the lower level solves a fuzzy programming problem with a constraint with respect to a satisfactory degree of the decision maker at the upper level.

Unfortunately, however, there is a possibility that the methods of Lai [2] and Shih et al. [3] lead a final solution to an undesirable one because of inconsistency between the fuzzy goals of the objective function and those of the decision variables. In order to overcome the problem in their methods, by eliminating the fuzzy goals for the decision variables, Sakawa et al. have proposed interactive fuzzy programming for two-level or multi-level linear programming problems to obtain a satisfactory solution for decision makers [4,5]. Extensions to two-level linear fractional programming problems [5] and decentralized two-level linear programming problems [6-8] have also been considered. The subsequent works on two-level or multi-level programming have been appearing [9-12]. A recent survey paper of Sakawa and Nishizaki [13] is devoted to reviewing and classifying the numerous major papers in the area of so-called cooperative multi-level programming.

In actual decision making situations, however, we must often make a decision on the basis of vague information or uncertain data. For such decision making problems involving uncertainty, there exist two typical approaches: probability theoretic approach [14-16] and fuzzy-theoretic one [16-19]. Stochastic programming, as an optimization method based on the probability theory, have been developing in various ways [14-16], including two stage problems considered by Dantzig [20] and chance constrained programming proposed by Charnes et al. [21]. Fuzzy mathematical programming representing the vagueness in decision making situations by fuzzy 
concepts have been studied by many researchers $[17,18]$. Fuzzy multiobjective linear programming, first proposed by Zimmermann [17], have been also developed by numerous researchers, and an increasing number of successful applications has been appearing [18,19,22,23]. In particular, after reformulating stochastic multiobjective linear programming problems using several models for chance constrained programming, Sakawa et al. [24-26] presented an interactive fuzzy satisficing method to derive a satisficing solution for the $\mathrm{DM}$ as a generalization of their previous results [18, 22, 23, 27-30].

Furthermore, in real world decision making situations, it is often found that decision variables in a multiobjective stochastic programming problem the formulated programming problems are not continuous but rather discrete. To deal with practical sizes of the stochastic multi-level 0-1 programming problems formulated for decision making problems in the real world, an efficient tabu search method for general 0-1 programming problems is introduced.

Under these circumstances, in this paper, through a simultaneous consideration of both random variables and integer decision variables involved in the real world hierarchical decision making problems, we first formulate multi-level 0-1 programming problems with random variable coefficients in both objective functions and constraints. The main contribution of this paper is to provide a novel decision making methodology including a new model, solution concept and solution algorithm to deal with more realistic problems in the real world, by simultaneously considering various concepts such as hierarchy structure, fuzziness, randomness, 0-1 decision variables and interactive fuzzy programming, while most of previous papers dealt with either of the concepts or a part of them.

By employing chance constrained programming [31], stochastic constraints are transformed into deterministic ones. Adopting the fractile criterion optimization model $[32,33]$, the minimization of each stochastic objective function is replaced with the optimization of the target variables under the condition that the attained probabilities are greater than or equal to certain permissible levels. Under some appropriate assumptions for distribution functions, the formulated stochastic multi-level 0-1 programming problems are transformed into deterministic ones. In our interactive method, after determining the fuzzy goals of the DMs at all levels, a satisfactory solution is derived efficiently by updating the satisfactory degrees of the DMs at the upper level with considerations of overall satisfactory balance among all levels. For solving the transformed deterministic problems efficiently, we also propose a novel tabu search method by extending tabu search based on strategic oscillation for multidimensional 0-1 knapsack problems [34] into general 0-1 programming problems.

\section{Problem formulation with frac- tile models}

Consider stochastic multi-level 0-1 programming problems where each of the DMs at all levels takes overall satisfactory balance among all levels into considera- tion and tries to optimize each objective function. Such a stochastic multi-level 0-1 programming problem can be formulated as

$$
\left.\begin{array}{cl}
\underset{\text { DM1 (Level 1) }}{\operatorname{minimize}} & z_{1}(\boldsymbol{x})=\boldsymbol{c}_{11}(\omega) \boldsymbol{x}_{1}+\cdots+\boldsymbol{c}_{1 K}(\omega) \boldsymbol{x}_{K} \\
\vdots & \vdots \\
\text { DMinimize } & z_{K}(\boldsymbol{x})=\boldsymbol{c}_{K 1}(\omega) \boldsymbol{x}_{1}+\cdots+\boldsymbol{c}_{K K}(\omega) \boldsymbol{x}_{K} \\
\text { subject to } & A_{1} \boldsymbol{x}_{1}+\cdots+A_{K} \boldsymbol{x}_{K} \leq \boldsymbol{b}(\omega) \\
& \boldsymbol{x}_{1} \in\{0,1\}^{n_{1}}, \ldots, \boldsymbol{x}_{K} \in\{0,1\}^{n_{K}}
\end{array}\right\}
$$

where $\boldsymbol{x}_{l}, l=1, \ldots, K$, is an $n_{l}$-dimensional 0-1 decision variable column vector, $A_{l}, l=1, \ldots, K$ are $m \times n_{l}$ coefficient matrices, and $\boldsymbol{c}_{l j}(\omega), l=1, \ldots, K, j=1, \ldots, K$ are $n_{j}$-dimensional Gaussian random variable row vectors with mean vectors $\overline{\boldsymbol{c}}_{l j}$, and covariance matrices $V_{l p q}=\left(v_{h_{p} h_{q}}^{l}\right)=\left(\operatorname{Cov}\left[c_{l h_{p}}(\omega), c_{l h_{q}}(\omega)\right]\right), p=1, \ldots, K$, $q=1, \ldots, K$, and they are independent of each other, and $\boldsymbol{b}(\omega)$ is a random variable vector whose joint distribution function is $F(\cdot)$.

Since (1) contains random variable coefficients, solution methods for ordinary mathematical programming problems cannot be applied directly. Consequently, we first deal with the constraints in (1) as chance constraints [21] which mean that the constraints need to be satisfied with a certain probability (satisficing level) and over. Namely, replacing constraints in (1) by chance constraints with a satisficing level $\boldsymbol{\beta}$, the problem can be transformed as:

$$
\begin{array}{cc}
\underset{\mathrm{DM}(\text { Level 1) }}{\operatorname{minimize}} & z_{1}(\boldsymbol{x})=\boldsymbol{c}_{11}(\omega) \boldsymbol{x}_{1}+\cdots+\boldsymbol{c}_{1 K}(\omega) \boldsymbol{x}_{K} \\
\vdots & \vdots \\
\underset{\mathrm{DMK}(\text { Level } K)}{\operatorname{minimize}} & z_{K}(\boldsymbol{x})=\boldsymbol{c}_{K 1}(\omega) \boldsymbol{x}_{1}+\cdots+\boldsymbol{c}_{K K}(\omega) \boldsymbol{x}_{K} \\
\text { subject to } & \operatorname{Pr}\left\{\boldsymbol{a}_{i 1} \boldsymbol{x}_{1}+\cdots+\boldsymbol{a}_{i K} \boldsymbol{x}_{K} \leq b_{i}(\omega)\right\} \\
& \geq \beta_{i}, i=1, \ldots, m \\
& \boldsymbol{x}_{1} \in\{0,1\}^{n_{1}}, \ldots, \boldsymbol{x}_{K} \in\{0,1\}^{n_{K}}
\end{array}
$$

where $\boldsymbol{a}_{i j}$ is the $i$ th row vector of $A_{l}, l=1, \ldots, K$, and $b_{i}(\omega)$ is the $i$ th element of $\boldsymbol{b}(\omega)$.

The first constraint in (2) is rewritten as:

$$
\begin{array}{ll} 
& \operatorname{Pr}\left\{\boldsymbol{a}_{i 1} \boldsymbol{x}_{1}+\cdots+\boldsymbol{a}_{i K} \boldsymbol{x}_{K} \leq b_{i}(\omega)\right\} \geq \beta_{i} \\
\Leftrightarrow & 1-\operatorname{Pr}\left\{\boldsymbol{a}_{i 1} \boldsymbol{x}_{1}+\cdots+\boldsymbol{a}_{i K} \boldsymbol{x}_{K} \geq b_{i}(\omega)\right\} \geq \beta_{i} \\
\Leftrightarrow & 1-F_{i}\left(\boldsymbol{a}_{i 1} \boldsymbol{x}_{1}+\cdots+\boldsymbol{a}_{i K} \boldsymbol{x}_{K}\right) \geq \beta_{i} \\
\Leftrightarrow & F_{i}\left(\boldsymbol{a}_{i 1} \boldsymbol{x}_{1}+\cdots+\boldsymbol{a}_{i K} \boldsymbol{x}_{K}\right) \leq 1-\beta_{i} \\
\Leftrightarrow & \boldsymbol{a}_{i 1} \boldsymbol{x}_{1}+\cdots+\boldsymbol{a}_{i K} \boldsymbol{x}_{K} \leq F_{i}^{*}\left(1-\beta_{i}\right)
\end{array}
$$

where $F_{i}^{*}$ is a pseudo-inverse function of $F_{i}$.

Letting $\hat{b}_{i}=F_{i}^{*}\left(1-\beta_{i}\right)$, problem (2) can be rewritten as:

$$
\begin{array}{cc}
\underset{\mathrm{DM1}(\text { Level 1) }}{\operatorname{minimize}} & z_{1}(\boldsymbol{x})=\boldsymbol{c}_{11}(\omega) \boldsymbol{x}_{1}+\cdots+\boldsymbol{c}_{1 K}(\omega) \boldsymbol{x}_{K} \\
\vdots & \vdots \\
\underset{\mathrm{DM} K(\text { Level } K)}{\operatorname{minimize}} & z_{K}(\boldsymbol{x})=\boldsymbol{c}_{K 1}(\omega) \boldsymbol{x}_{1}+\cdots+\boldsymbol{c}_{K K}(\omega) \boldsymbol{x}_{K} \\
\text { subject to } & A_{1} \boldsymbol{x}_{1}+\cdots+A_{K} \boldsymbol{x}_{K} \leq \hat{\boldsymbol{b}} \\
& \boldsymbol{x}_{1} \in\{0,1\}^{n_{1}}, \ldots, \boldsymbol{x}_{K} \in\{0,1\}^{n_{K}}
\end{array}
$$

where $\hat{\boldsymbol{b}}=\left(\hat{b}_{1}, \hat{b}_{2}, \ldots, \hat{b}_{m}\right)^{T}$.

It should be noted here that the constraint of (3) is no longer stochastic but becomes deterministic through the idea of chance constraint. 
Charnes and Cooper [31] also considered three types of decision rules for optimizing objective functions with random variables: (i) the minimum or maximum expected value model, (ii) the minimum variance model, and (iii) the maximum probability model, which are referred to as the expectation model, the variance model, and the probability model, respectively. Moreover, Kataoka [32] and Geoffrion [33] individually proposed the fractile model.

For instance, let the objective function represent a profit. If the DM wishes to simply maximize the expected profit without caring about the fluctuation of the profit, the expectation model to optimize the expectation of the objective function is appropriate. On the other hand, if the DM hopes to decrease the fluctuation of the profit as little as possible from the viewpoint of the stability of the profit, the variance model to minimize the variance of the objective function is useful. In contrast to these two types of optimizing approaches, as satisficing approaches, the probability model and the fractile model have been proposed.

When the DM wants to maximize the probability that the profit is greater than or equal to a certain permissible level, probability model is recommended. In contrast, when the DM wishes to optimize such a permissible level under a given threshold probability with respect to the achieved profit, the fractile model will be appropriate. In this paper, assuming that the DM is interested in the probability that each objective function attains a goal value rather than the expectation or variance of each membership function, we adopt the fractile criterion optimization as a decision making model.

In the fractile criterion optimization model, the minimization of each of objective function $z_{l}(\boldsymbol{x})$ in (3) is substituted with the optimization of the target value that $z_{l}(\boldsymbol{x})$ is less than or equal to a certain permissible level $f_{l}$ under the chance constraints. Through fractile criteria optimization, problem (3) can be rewritten as:

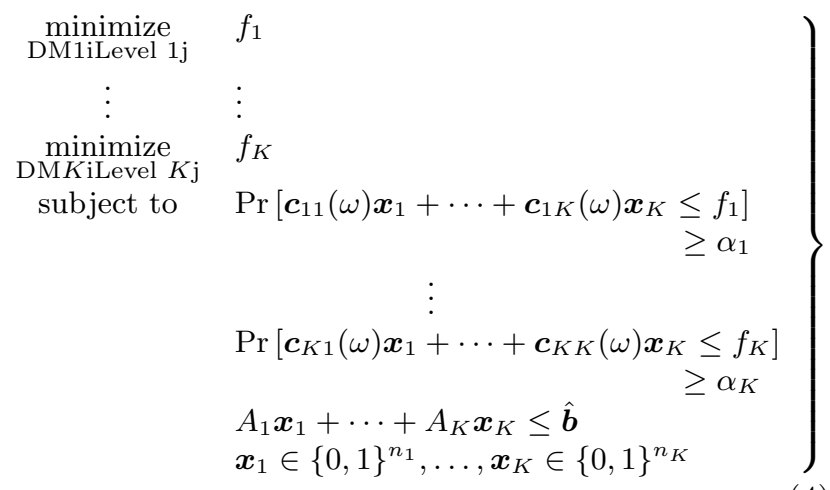

Since all elements of $\boldsymbol{c}_{l j}(\omega), l=1, \ldots, K, j=1, \ldots, K$ are assumed to be Gaussian random variable with mean $\bar{c}_{l j}$ and covariance matrices $V_{l p q}, p=1, \ldots, K, q=$ $1, \ldots, K$.

Then, $\quad\left(h_{l} \quad-\quad\left(\bar{c}_{l 1} \boldsymbol{x}_{1}+\cdots+\right.\right.$ $\left.\left.\bar{c}_{l K} \boldsymbol{x}_{K}\right)\right) / \sqrt{\left(\boldsymbol{x}_{1}^{T}, \ldots, \boldsymbol{x}_{K}^{T}\right) V_{l}\left(\boldsymbol{x}_{1}^{T}, \ldots, \boldsymbol{x}_{K}^{T}\right)^{T}}$ is a random variable following the standard Gaussian distribution with mean 0 and variance 1 . Hence, we can rewrite the objective functions in (4) as follows.

$$
\begin{aligned}
& \operatorname{Pr}\left\{z_{l}\left(\boldsymbol{x}_{1}, \ldots, \boldsymbol{x}_{K}, \omega\right) \leq f_{l}\right\} \\
& =\operatorname{Pr}\left\{\boldsymbol{c}_{l 1}(\omega) \boldsymbol{x}_{1}+\cdots+\boldsymbol{c}_{l K}(\omega) \boldsymbol{x}_{K} \leq f_{l}\right\} \\
& =\operatorname{Pr}\left\{\frac{\boldsymbol{c}_{l 1}(\omega) \boldsymbol{x}_{1}+\cdots+\boldsymbol{c}_{l K}(\omega) \boldsymbol{x}_{K}-\left(\bar{c}_{l 1} \boldsymbol{x}_{1}+\cdots+\bar{c}_{l K} \boldsymbol{x}_{K}\right)}{\sqrt{\left(\boldsymbol{x}_{1}^{T}, \ldots, \boldsymbol{x}_{K}^{T}\right) V_{l}\left(\boldsymbol{x}_{1}^{T}, \ldots, \boldsymbol{x}_{K}^{T}\right)^{T}}}\right. \\
& \left.\quad \leq \frac{f_{l}-\left(\bar{c}_{l 1} \boldsymbol{x}_{1}+\cdots+\bar{c}_{l K} \boldsymbol{x}_{K}\right)}{\sqrt{\left(\boldsymbol{x}_{1}^{T}, \ldots, \boldsymbol{x}_{K}^{T}\right) V_{l}\left(\boldsymbol{x}_{1}^{T}, \ldots, \boldsymbol{x}_{K}^{T}\right)^{T}}}\right\} \\
& =\phi_{l}\left(\frac{f_{l}-\left(\bar{c}_{l 1} \boldsymbol{x}_{1}+\cdots+\bar{c}_{l K} \boldsymbol{x}_{K}\right)}{\sqrt{\left(\boldsymbol{x}_{1}^{T}, \ldots, \boldsymbol{x}_{K}^{T}\right) V_{l}\left(\boldsymbol{x}_{1}^{T}, \ldots, \boldsymbol{x}_{K}^{T}\right)^{T}}}\right) \geq \alpha_{l},
\end{aligned}
$$

where $\phi_{l}(\cdot)$ is the distribution function of the standard Gaussian random variable. From the monotonicity of the distribution function, we can define the inverse function $\phi_{l}^{-1}(\cdot)$ of $\phi_{l}(\cdot)$. Then, the above inequalities are expressed as:

$$
\begin{aligned}
& \frac{f_{l}-\left(\overline{\boldsymbol{c}}_{l 1} \boldsymbol{x}_{1}+\cdots+\overline{\boldsymbol{c}}_{l K} \boldsymbol{x}_{K}\right)}{\sqrt{\left(\boldsymbol{x}_{1}^{T}, \ldots, \boldsymbol{x}_{K}^{T}\right) V_{l}\left(\boldsymbol{x}_{1}^{T}, \ldots, \boldsymbol{x}_{K}^{T}\right)^{T}}} \geq K_{\alpha_{l}} \\
& \Leftrightarrow \quad f_{l} \geq\left(\overline{\boldsymbol{c}}_{l 1} \boldsymbol{x}_{1}+\cdots+\overline{\boldsymbol{c}}_{l K} \boldsymbol{x}_{K}\right) \\
& +K_{\alpha_{l}} \sqrt{\left(\boldsymbol{x}_{1}^{T}, \ldots, \boldsymbol{x}_{K}^{T}\right) V_{l}\left(\boldsymbol{x}_{1}^{T}, \ldots, \boldsymbol{x}_{K}^{T}\right)^{T}}
\end{aligned}
$$

Letting $K_{\alpha_{l}}=\phi_{l}^{-1}\left(\alpha_{l}\right), l=1, \cdots, K$ and noting that the equality

$$
\begin{aligned}
f_{l}= & \left(\overline{\boldsymbol{c}}_{l 1} \boldsymbol{x}_{1}+\cdots+\overline{\boldsymbol{c}}_{l K} \boldsymbol{x}_{K}\right) \\
& +K_{\alpha_{l}} \sqrt{\left(\boldsymbol{x}_{1}^{T}, \ldots, \boldsymbol{x}_{K}^{T}\right) V_{l}\left(\boldsymbol{x}_{1}^{T}, \ldots, \boldsymbol{x}_{K}^{T}\right)^{T}}
\end{aligned}
$$

holds at the minimum of $f_{l}$, problem (4) is equivalent to the following deterministic multi-level programming problem:

$$
\left.\begin{array}{cc}
\underset{\text { DM1iLevel 1j }}{\operatorname{minimize}} & Z_{1}^{F}\left(\boldsymbol{x}_{1}, \ldots, \boldsymbol{x}_{K}\right) \\
= & \left(\overline{\boldsymbol{c}}_{11} \boldsymbol{x}_{1}+\cdots+\overline{\boldsymbol{c}}_{1 K} \boldsymbol{x}_{K}\right) \\
& +K_{\alpha_{1}} \sqrt{\left(\boldsymbol{x}_{1}^{T}, \ldots, \boldsymbol{x}_{K}^{T}\right) V_{1}\left(\boldsymbol{x}_{1}^{T}, \ldots, \boldsymbol{x}_{K}^{T}\right)^{T}} \\
\vdots & \vdots \\
\operatorname{DMKiLevel~} K \mathrm{j}^{\operatorname{minimize}} & Z_{K}^{F}\left(\boldsymbol{x}_{1}, \ldots, \boldsymbol{x}_{K}\right) \\
& =\left(\overline{\boldsymbol{c}}_{K 1} \boldsymbol{x}_{1}+\cdots+\overline{\boldsymbol{c}}_{K K} \boldsymbol{x}_{K}\right) \\
& +K_{\alpha_{K}} \sqrt{\left(\boldsymbol{x}_{1}^{T}, \ldots, \boldsymbol{x}_{K}^{T}\right) V_{K}\left(\boldsymbol{x}_{1}^{T}, \ldots, \boldsymbol{x}_{K}^{T}\right)^{T}}
\end{array}\right\}
$$

where $X$ denotes the feasible region satisfying the constraints of (4).

\section{Interactive fuzzy programming}

In the deterministic multi-level $0-1$ programming problem (5), considering the vague nature of the DMs' judgments for the target values $Z_{l}^{F}(\boldsymbol{x}), l=1,2, \ldots, K$ it seems natural to assume that the DMs have fuzzy goals such as " $Z_{l}^{F}(\boldsymbol{x})$ should be substantially greater than or equal to some specific value."

Through the introduction of a membership function $\mu_{l}(\cdot)$ for quantifying a fuzzy goal for the $l$ th objective function in (5), (5) can be rewritten as:

$$
\left.\begin{array}{cc}
\underset{\text { DM1iLevel } 1 \mathrm{j}}{\operatorname{maximize}} & \mu_{1}\left(Z_{1}^{F}\left(\boldsymbol{x}_{1}, \ldots, \boldsymbol{x}_{K}\right)\right) \\
\vdots & \vdots \\
\underset{\text { DM } K \text { iLevel } K \mathrm{j}}{\operatorname{maximize}} & \mu_{K}\left(Z_{K}^{F}\left(\boldsymbol{x}_{1}, \ldots, \boldsymbol{x}_{K}\right)\right) \\
\text { subject to } & \boldsymbol{x} \in X
\end{array}\right\}
$$


where $\mu_{l}(\cdot)$ is assumed to be nondecreasing.

Although the membership function does not always need to be linear, for the sake of simplicity, we adopt a linear membership function. To be more specific, if the DM feels that $Z_{l}^{F}(\boldsymbol{x})$ should be greater than or equal to at least $Z_{l, 0}^{F}$ and $Z_{l}^{F}(\boldsymbol{x}) \geq Z_{l, 1}^{F}\left(>Z_{l, 0}^{F}\right)$ is satisfactory, the linear membership function $\mu_{l}\left(Z_{l}^{F}(\boldsymbol{x})\right)$ is defined as:

$$
=\left\{\begin{array}{cl}
\mu_{l}\left(Z_{l}^{F}(\boldsymbol{x})\right) & \mu_{l}\left(Z_{l}^{F}(\boldsymbol{x})\right)<Z_{l, 0}^{F} \\
\frac{\mu_{l}\left(Z_{l}^{F}(\boldsymbol{x})\right)-Z_{l, 0}^{F}}{Z_{l, 1}^{F}-Z_{l, 0}^{F}}, & Z_{l, 0}^{F} \leq \mu_{l}\left(Z_{l}^{F}(\boldsymbol{x})\right) \leq Z_{l, 1}^{F} \\
1, & \mu_{l}\left(Z_{l}^{F}(\boldsymbol{x})\right)>Z_{l, 1}^{F}
\end{array}\right.
$$

and it is depicted in Figure 1.

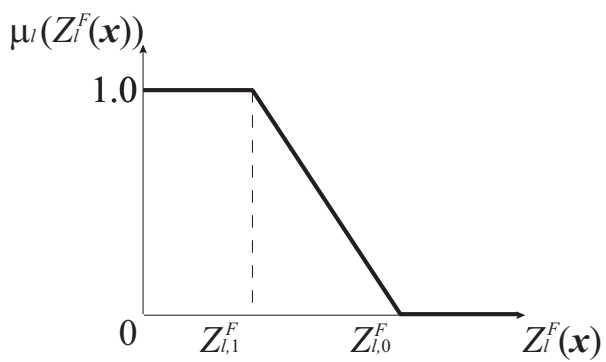

Figure 1. Linear membership function.

Zimmermann [17] suggested a method for assessing the parameter values of the linear membership function. In his method, the parameter values $Z_{l, 1}^{F}, l=1, \ldots, K$ are determined as

$$
\begin{aligned}
Z_{1,1}^{F} & =Z_{1, \max }^{F} \\
& =Z_{1}^{F}\left(\boldsymbol{x}_{1, \max }^{1}, \ldots, \boldsymbol{x}_{K, \max }^{1}\right) \\
& =\underset{\left(\boldsymbol{x}_{1}^{T}, \ldots, \boldsymbol{x}_{K}^{T}\right)^{T} \in X}{\max _{1}^{F}\left(\boldsymbol{x}_{1}, \ldots, \boldsymbol{x}_{K}\right)} \\
& \vdots \\
Z_{K, 1}^{F} & =Z_{K, \max }^{F} \\
& =Z_{K}^{F}\left(\boldsymbol{x}_{1, \max }^{K}, \ldots, \boldsymbol{x}_{K, \max }^{K}\right) \\
& =\underset{\left(\boldsymbol{x}_{1}^{T}, \ldots, \boldsymbol{x}_{K}^{T}\right)^{T} \in X}{\max _{K}\left(\boldsymbol{x}_{1}, \ldots, \boldsymbol{x}_{K}\right)}
\end{aligned}
$$

and the parameter values $X_{l, 0}^{F}, l=1, \ldots, K$ are specified as

$$
\begin{gathered}
Z_{1,0}^{F}=Z_{1}^{F}\left(\boldsymbol{x}_{1, \min }^{1}, \ldots, \boldsymbol{x}_{K, \min }^{1}\right) \\
\vdots \\
Z_{K, 0}^{F}=Z_{K}^{F}\left(\boldsymbol{x}_{1, \min }^{K}, \ldots, \boldsymbol{x}_{K, \min }^{K}\right)
\end{gathered}
$$

where $\boldsymbol{x}_{l, \text { min }}=\left(\boldsymbol{x}_{l, \text { min }}^{1}, \ldots, \boldsymbol{x}_{l, \text { min }}^{K}\right), l=1, \ldots, K$ is an optimal solution to the following problem

$$
\left.\begin{array}{rl}
\operatorname{maximize} & Z_{l}^{F}\left(\boldsymbol{x}_{1}, \ldots, \boldsymbol{x}_{K}\right) \\
& =\phi_{l}\left(\frac{f_{l}-\left(\bar{c}_{l 1} \boldsymbol{x}_{1}+\cdots+\bar{c}_{l K} \boldsymbol{x}_{K}\right)}{\sqrt{\left(\boldsymbol{x}_{1}^{T}, \ldots, \boldsymbol{x}_{K}^{T}\right) V_{l}\left(\boldsymbol{x}_{1}^{T}, \ldots, \boldsymbol{x}_{K}^{T}\right)^{T}}}\right) \\
\text { subject to } & \boldsymbol{x} \in X
\end{array}\right\} .
$$

Since (8) is a 0-1 programming problem, it can be solved by tabu search based on strategic oscillation [34].

Then, by setting the parameters as described above, the linear membership functions (7) is identified.

To derive an overall satisfactory solution to the membership function maximization 6 , we first find the maximizing decision of the fuzzy decision proposed by Bellman and Zadeh [35]. Namely, the following problem is solved for obtaining a solution which maximizes the smaller degree of satisfaction between those of all DMs:

$$
\underset{\boldsymbol{x} \in X}{\operatorname{maximize}} \min _{l=1, \ldots, K}\left\{\mu_{l}\left(Z_{l}^{F}(\boldsymbol{x})\right)\right\}
$$

Solving problem (9), we can obtain a solution which maximizes the smaller satisfactory degree between those of all DMs.

\section{Termination conditions of the interactive process}

(1) For all $\lambda=1, \ldots, K-1, \mathrm{DM} \lambda$ 's satisfactory degree is larger than or equal to the minimal satisfactory level $\hat{\delta}_{\Delta_{\lambda}}$ specified by $\operatorname{DM} \lambda$.

(2) For all $\lambda=1, \ldots, K-1$, the ratio $\delta_{\lambda}$ of satisfactory degrees is in the closed interval the lower and the upper bounds of which are specified by $\operatorname{DM} \lambda$.

Condition (1) means DM $\lambda$ 's required condition for solutions proposed by $\operatorname{DM}(\lambda+1)$. Condition (2) is provided in order to keep overal satisfactory balance among all the level.

Unless the conditions are satisfied simultaneously, $\operatorname{DM} \lambda, \lambda=1, \ldots, K$, needs to update the minimal satisfactory level $\hat{\delta}_{\Delta_{\lambda}}$. Suppose that the DMs from at the $(q+1)$ th level to at the $(K-1)$ th level, i.e., $\operatorname{DM}(q+1)$, $\operatorname{DM}(q+2), \ldots$, and $\operatorname{DM}(K-1)$, satisfy the proposed solution but $\mathrm{DM} q$ does not satisfy it. Then $\mathrm{DM} q$, $\operatorname{DM}(q+1), \ldots$, and $\operatorname{DM}(K-1)$ need to update their minimal satisfactory levels $\hat{\delta}_{\Delta_{\lambda}}, \lambda=q, q+1, \ldots, K-1$. For any two levels adjacent to each other, giving a DM at an upper level serious consideration, a DM at a lower level should update the minimal satisfactory level.

Now we are ready to propose interactive fuzzy programming for deriving a satisfactory solution by updating the satisfactory degree of the DM at the upper level with considerations of overall satisfactory balance among all the levels.

$\underline{\text { Computational procedure of interactive fuzzy programming }}$

Step 1: Ask the decision maker at the upper level, DM1, to subjectively determine a satisficing levels $\alpha_{l} \in(0,1), l=1,2, \ldots, K$ for objective functions and $\beta_{i} \in(0,1), i=1,2, \ldots, m$ for constraints. Go to Step 2.

Step 2: The following problems are solved to find the minimum values $Z_{l, \min }^{F}=Z_{l}^{F}\left(\boldsymbol{x}^{l, \min }\right)$ and $Z_{l, M}^{F}=$ $\max _{j=1, \ldots, K}\left\{Z_{l}^{F}\left(\boldsymbol{x}^{j, \min }\right)\right\}$ of objective functions $Z_{l}^{F}(\boldsymbol{x})$ under the chance constraints with satisficing levels $\alpha_{l}, l=1,2, \ldots, K$ and $\beta_{i}, i=1,2, \ldots, m$.

$$
\left.\begin{array}{rl}
\operatorname{minimize} & Z_{l}^{F}\left(\boldsymbol{x}_{1}, \ldots, \boldsymbol{x}_{K}\right) \\
\text { subject to } & \boldsymbol{x} \in X
\end{array}\right\}, l=1, \ldots, K
$$

If the set of feasible solutions to these problems is empty, the satisficing levels $\alpha_{l} \in(0,1), l=$ $1,2, \ldots, K$ and $\beta_{i}, i=1,2, \ldots, m$ must be reassessed and return to step 1 . Otherwise, let $z_{l, \text { min }}^{F}$ be optimal objective function values to (10). Since (10) are 0-1 programming problems, they can be 
solved by tabu search based on strategic oscillation. Then, identify the linear membership function $\mu_{l}\left(Z_{l}^{F}(\boldsymbol{x})\right), l=1,2, \ldots, K$ of the fuzzy goal for the corresponding objective function. Go to Step 3.

Step 3: Solve the following corresponding maxmin problem.

$$
\underset{\boldsymbol{x} \in X}{\operatorname{maximize}} \min _{l=1, \ldots, K}\left\{\mu_{l}\left(Z_{l}^{F}(\boldsymbol{x})\right)\right\}
$$

Go to step 4.

Step 4: Ask DM1 to subjectively set the minimal satisfactory level $\hat{\delta}_{1}$. Then, solve the following maxmin problem.

$$
\left.\begin{array}{ll}
\underset{\boldsymbol{x} \in X}{\operatorname{maximize}} & \min _{l=2, \ldots, K}\left\{\mu_{l}\left(Z_{l}^{F}(\boldsymbol{x})\right)\right\} \\
\text { subject to } & \mu_{1}\left(p_{1}(\boldsymbol{x})\right) \geq \hat{\delta}_{1}
\end{array}\right\}
$$

Set $\lambda:=2 \mathrm{C} \lambda^{\prime}:=1$. Go to step 5.

Step 5: Ask DM $\lambda$ to set the membership function $\mu_{\Delta_{\lambda}}\left(\Delta_{\lambda}(\boldsymbol{x})\right)$ for the ratio $\Delta_{\lambda}=$ $\left(\mu_{\lambda+1}\left(Z_{\lambda+1}^{F}(\boldsymbol{x})\right)\right) /\left(\mu_{\lambda}\left(Z_{\lambda}^{F}(\boldsymbol{x})\right)\right)$ of satisfactory degrees and the minimal satisfactory level $\hat{\delta}_{\Delta_{\lambda}}$. Solve the following maxmin problem.

$$
\left.\begin{array}{cl}
\underset{\boldsymbol{x} \in X}{\operatorname{maximize}} & \min _{l=\lambda+1, \ldots, K}\left\{\mu_{l}\left(Z_{l}^{F}(\boldsymbol{x})\right)\right\} \\
\text { subject to } & \mu_{1}\left(Z_{1}^{F}(\boldsymbol{x})\right) \geq \hat{\delta}_{1} \\
& \mu_{\Delta_{2}}\left(\Delta_{2}(\boldsymbol{x})\right) \geq \hat{\delta}_{\Delta_{2}} \\
& \vdots \\
& \mu_{\Delta_{\lambda}}\left(\Delta_{\lambda}(\boldsymbol{x})\right) \geq \hat{\delta}_{\Delta_{\lambda}}
\end{array}\right\}
$$

Repeat this step until $\lambda=K-1$.

Step 6: If the current solution satisfies the termination conditions, DM $K-\lambda^{\prime}$ accepts it, and $K-\lambda^{\prime}=1$, then the procedure stops and the current solution is determined to be a satisfactory solution. Otherwise, ask DM $K-\lambda^{\prime}$ to update the minimal satisfactory level $\hat{\delta}_{\Delta_{K-\lambda^{\prime}}}$. If $K-\lambda^{\prime}=1$, ask DM1 to update the minimal satisfactory level $\hat{\delta}_{1}$. Go to step 7 .

Step 7: Solve the following problem, and return to step 5.

$$
\left.\begin{array}{rl}
\operatorname{maximize} & v \\
\text { subject to } & \boldsymbol{x} \in X \\
& 0 \leq v \leq 1 \\
& \mu_{1}\left(Z_{1}^{F}(\boldsymbol{x})\right) \geq \hat{\delta}_{1} \\
& \mu_{\Delta_{2}}\left(\Delta_{2}(\boldsymbol{x})\right) \geq \hat{\delta}_{\Delta_{2}} \\
& \quad \vdots \\
& \mu_{\Delta_{K-1}}\left(\Delta_{K-1}(\boldsymbol{x})\right) \geq \hat{\delta}_{\Delta_{K-1}} \\
& \Pi_{l=K-\lambda^{\prime}+1}^{K} \hat{\Delta}_{l} \mu_{K-\lambda^{\prime}+1}\left(Z_{K-\lambda^{\prime}+1}^{F}(\boldsymbol{x})\right) \geq v \\
& \vdots \\
& \hat{\Delta}_{K-1} \hat{\hat{\Delta}}_{K-2} \mu_{K-2}\left(Z_{K-2}^{F}(\boldsymbol{x})\right) \geq v \\
& \hat{\Delta}_{K-1} \mu_{K-1}\left(Z_{K-1}^{F}(\boldsymbol{x})\right) \geq v \\
& \mu_{K}\left(Z_{K}^{F}(\boldsymbol{x})\right) \geq v
\end{array}\right\}
$$

It should be noted here that, in the proposed interactive fuzzy programming method, it is required to solve the 0-1 programming problems (10), (11), (12), (13) and (14), which is apparently difficult to solve.

\section{Tabu search for general 0-1 programming problems}

For solving the $0-1$ programming problems in the proposed interactive fuzzy programming method, it is constructive to extend tabu search based on strategic oscillation for multidimensional 0-1 knapsack problems [34] into general 0-1 programming problems.

With this observation, consider a general 0-1 programming problem formulated as:

$$
\left.\begin{array}{rl}
\operatorname{minimize} & f(\boldsymbol{x}) \\
\text { subject to } & g_{i}(\boldsymbol{x}) \leq 0, i=1, \ldots, m \\
& \boldsymbol{x} \in\{0,1\}^{n}
\end{array}\right\}
$$

where $f(\cdot)$ and $g_{i}(\cdot), i=1, \ldots, m$ are convex or nonconvex real-valued functions and $\boldsymbol{x}=\left(x_{1}, \ldots, x_{n}\right)^{T}$ is an $n$-dimensional column vector of $0-1$ decision variables.

The tabu search proposed in [34] made use of the property of multidimensional 0-1 knapsack problems that the improvement or disimprovement of the objective function value corresponds with the decrease or increase of the degree of feasibility. From the property, it is clear that the optimal solution to multidimensional 0-1 knapsack problems exists in the area near the boundary of the feasible region which is called the promising zone. Thus, the search direction in multidimensional 0-1 knapsack problems can be controlled by checking the change of the objective function value. In the case of general 01 programming problems, observing that the monotone relation between the objective function value and the degree of feasibility no longer holds, the promising zone does not always exist near the boundary of the feasible region. Considering that the promising zone originally means the area which include an optimal solution, we define the promising zone for general 0-1 programming problems as neighborhoods of local optimal solutions. Thus, in order to use not only the change of the objective function value but the degree of feasibility, we introduce the index of surplus of constraints $\delta(\boldsymbol{x})$ and that of slackness of constraints $\epsilon(\boldsymbol{x})$ defined as:

$$
\begin{gathered}
\delta(\boldsymbol{x})=\sum_{i \in I^{+}} \delta_{i}(\boldsymbol{x})=\sum_{i \in I^{+}} g_{i}(\boldsymbol{x}) \\
\epsilon(\boldsymbol{x})=\sum_{i \in I^{-}} \epsilon_{i}(\boldsymbol{x})=\sum_{i \in I^{-}}-g_{i}(\boldsymbol{x})
\end{gathered}
$$

where $I^{+}=\left\{i \mid g_{i}(\boldsymbol{x})>0, i \in\{1, \ldots, m\}\right\}$ and $I^{-}=\left\{i \mid g_{i}(\boldsymbol{x})<0, i \in\{1, \ldots, m\}\right\}$, Furthermore, let $\Delta_{j} f(\boldsymbol{x})$ denote the change of $f(\boldsymbol{x})$ by setting $x_{j}:=1-x_{j}$. Similarly, $\Delta_{j} \delta(\boldsymbol{x}), \Delta_{j} \delta_{i}(\boldsymbol{x}), \Delta_{j} \epsilon(\boldsymbol{x})$ and $\Delta_{j} \epsilon_{i}(\boldsymbol{x})$ are defined for $x_{j}:=1-x_{j}$. In addition, we assign the feasible solution to $\boldsymbol{x}^{\dagger}$, and update $\boldsymbol{x}^{\dagger}$ when the feasible solution is updated.

Computational procedure of tabu search for general 0-1 programming problems

\section{Step 0: INITIALIZATION}

Generate an initial solution $\boldsymbol{x}$ at random, and initialize the tabu list (TL). Set the tabu term (TT), the depth $(D)$, the maximum number of oscillation $\left(O_{\max }\right)$ and the oscillation counter $O:=1$. If $\boldsymbol{x}$ is feasible, go to step 4 . 
Otherwise, go to step 1.

\section{Step 1: TS_PROJECT}

The aim of this step is to move the current solution in the infeasible region to the promising zone in the gentlest ascent (disimproving) direction about the objective function with decreasing the surplus of constraints $\delta(\boldsymbol{x})$

While $\delta(\boldsymbol{x})$ is positive, i.e., the current solution is infeasible, repeat finding a non-tabu decision variable which decreases $\delta(\boldsymbol{x})$ and gives the lest disimprovement of the objective function value when its value would be changed, changing the value of the decision variable actually and adding the decision variable to TL. If there does not exist any non-tabu decision variable that decreases $\delta(\boldsymbol{x})$, select a decision variable randomly, change its value even if $\delta(\boldsymbol{x})$ increases and add the decision variable to TL. If $\delta(\boldsymbol{x})=0$ and there does not exist any decision variable which improves the objective function value by changing its value, go to step 2 .

\section{Step 2: TS_COMPLEMENT}

The aim of this step is to search the promising zone intensively.

Let $\boldsymbol{x}^{\prime}:=\boldsymbol{x}$ and $\boldsymbol{x}^{\prime \prime}:=\boldsymbol{x}^{\prime}$. Then, select several tabu decision variables of $\boldsymbol{x}^{\prime \prime}$ and change their values. If $\delta\left(\boldsymbol{x}^{\prime \prime}\right)=0$, then carry out step 4 . Otherwise, carry out step 1. If $f\left(\boldsymbol{x}^{\prime \prime}\right)<f(\boldsymbol{x})$ for the solution $\delta\left(\boldsymbol{x}^{\prime \prime}\right)$ obtained by step 4 or step 1 , let $\boldsymbol{x}:=\boldsymbol{x}^{\prime \prime}$. This procedure is repeated $D$ times. If the previous step of this step is step 1, then go to step 3. If the previous step of this step is step 4 , then go to step 5 .

\section{Step 3: TS_DROP}

The aim of this step is to move the current solution in the promising zone to the inside of the feasible region in the gentlest ascent direction of the objective function with increasing the slackness of constraints $\epsilon(\boldsymbol{x})$

Repeat finding a non-tabu decision variable which increases $\epsilon(\boldsymbol{x})$ and gives the lest disimprovement of the objective function when its value would be changed, changing the value of the decision variable actually and adding the decision variable to TL. If there does not exist any non-tabu decision variable that increases $\epsilon(\boldsymbol{x})$ or the number of repetitions of the above procedure exceeds TT, go to step 4.

\section{Step 4: TS_ADD}

The aim of this step is to move the current solution in the feasible region to the promising zone in the steepest descent (improving) direction about the objective function with keeping $\delta(\boldsymbol{x})=0$.

While $\delta(\boldsymbol{x})=0$, i.e., the current solution is feasible, repeat finding a non-tabu decision variable which keeps $\delta(\boldsymbol{x})=0$ and gives the greatest improvement of the objective function value when its value would be changed, changing the value of the decision variable actually and adding the decision variable to TL. If there does not exist such a decision variable, go to step 2.

\section{Step 5: TS_INFEASIBLE_ADD}

The aim of this step is to move the current solution in the promising zone to the infeasible region in the steepest descent (if exist) or the gentlest ascent direction about the objective function with decreasing the slackness of constraints $\epsilon(\boldsymbol{x})$ or increasing the surplus of constraints $\delta(\boldsymbol{x})$.

Repeat finding a non-tabu decision variable which decreasing the slackness of constraints $\epsilon(\boldsymbol{x})$ or increasing the surplus of constraints $\delta(\boldsymbol{x})$ and gives the greatest improvement (if exist) or the lest disimprovement of the objective function value when its value would be changed, changing the value of the decision variable actually and adding the decision variable to TL. If there does not exist such a decision variable or the number of repetitions of the above procedure exceeds $O_{\max }$, return to step 1 .

The search procedure of the proposed tabu search for general 0-1 programming problems is illustrated in Figure 2

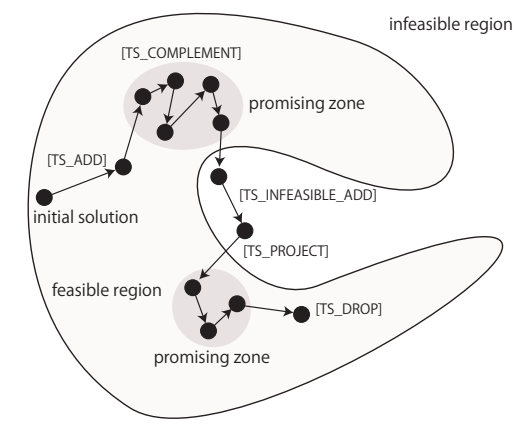

Figure 2. Tabu search for general 0-1 programming.

\section{$5 \quad$ Numerical example}

As an illustrative numerical example, consider the following stochastic three-level 0-1 programming problem:

$$
\left.\begin{array}{cl}
\underset{\text { DM1 (Level 1) }}{\operatorname{minimize}} & \boldsymbol{c}_{11}(\omega) \boldsymbol{x}_{1}+\boldsymbol{c}_{12}(\omega) \boldsymbol{x}_{2}+\boldsymbol{c}_{13}(\omega) \boldsymbol{x}_{3} \\
\text { minimize } & \boldsymbol{c}_{21}(\omega) \boldsymbol{x}_{1}+\boldsymbol{c}_{22}(\omega) \boldsymbol{x}_{2}+\boldsymbol{c}_{23}(\omega) \boldsymbol{x}_{3} \\
\text { DM2 (Level 2) } & \boldsymbol{c}_{31}(\omega) \boldsymbol{x}_{1}+\boldsymbol{c}_{32}(\omega) \boldsymbol{x}_{2}+\boldsymbol{c}_{33}(\omega) \boldsymbol{x}_{3} \\
\text { minimize } & \boldsymbol{c}_{31}(\text { Level 3) } \\
\text { subject to } & A_{1} \boldsymbol{x}_{1}+A_{2} \boldsymbol{x}_{2}+A_{3} \boldsymbol{x}_{3} \leq \boldsymbol{b}(\omega) \\
& \boldsymbol{x}_{1} \in\{0,1\}^{n_{1}}, \ldots, \boldsymbol{x}_{3} \in\{0,1\}^{n_{3}}
\end{array}\right\}
$$

where $\boldsymbol{x}_{1}=\left(x_{1}, \ldots, x_{15}\right)^{T}, \boldsymbol{x}_{2}=\left(x_{16}, \ldots, x_{30}\right)^{T}, \boldsymbol{x}_{3}=$ $\left(x_{31}, \ldots, x_{45}\right)^{T}$; each entry of 15 -dimensional row constant vectors $\boldsymbol{c}_{i j}, i, j=1,2,3$, and each entry of $3 \times 15$ coefficient matrices $A_{1}, A_{2}$, and $A_{3}$ are random

Through the use of this numerical example, it is now appropriate to illustrate the proposed interactive fuzzy programming method.

In step 1 of the interactive fuzzy programming, DM1 specifies satisficing levels $\alpha_{l}, l=1,2,3$ and $\beta_{i}, i=$ $1,2, \ldots, 9$ as:

$$
\begin{gathered}
\left(\alpha_{1}, \alpha_{2}, \alpha_{3}\right)^{T}=(0.75,0.75,0.75)^{T} \\
\left(\beta_{1}, \beta_{2}, \beta_{3}, \beta_{4}, \beta_{5}, \beta_{6}, \beta_{7}, \beta_{8}, \beta_{9}\right)^{T}= \\
(0.95,0.80,0.85,0.90,0.90,0.85,0.85,0.95,0.80)^{T} .
\end{gathered}
$$

For the specified satisficing levels $\alpha_{l}, l=1,2,3$ and $\beta_{i}, i=1,2, \ldots, 9$, in step 2 , minimal values $Z_{l, \text { min }}^{F}$ and $Z_{l, M}^{F}$ of objective functions $Z_{l}^{F}\left(\boldsymbol{x}_{1}, \boldsymbol{x}_{2}, \boldsymbol{x}_{3}\right)$ under the chance constraints are calculated. By considering these values, the DMs identify the linear membership function whose parameter values are determined by the Zimmermann method [17].

In step 3, the maxmin problem is solved. The obtain result is shown at the column labeled " 1 st" in Table 1.

In step 4, for the obtained optimal solution, then, the ratio of satisfactory degrees $\Delta_{1}$ is equal to 0.9659 . 
Table 1. Interaction process

\begin{tabular}{c||c|c|c|c}
\hline Interaction & 1st & 2nd & 3rd & 4th \\
\hline$\hat{\delta}_{1}$ & & 0.8000 & 0.8000 & 0.8500 \\
\hline$\hat{\delta}_{\Delta_{1}}$ & & & & 0.8000 \\
\hline$\hat{\delta}_{\Delta_{2}}$ & & & 0.8000 & \\
\hline$\mu_{1}\left(Z_{1}^{F}(\boldsymbol{x})\right)$ & 0.7495 & 0.8247 & 0.8010 & 0.8680 \\
\hline$\mu_{2}\left(Z_{2}^{F}(\boldsymbol{x})\right)$ & 0.7239 & 0.6677 & 0.7107 & 0.6778 \\
\hline$\mu_{3}\left(Z_{3}^{F}(\boldsymbol{x})\right)$ & 0.7065 & 0.6925 & 0.6311 & 0.6162 \\
\hline$\Delta_{1}(\boldsymbol{x})$ & 0.9659 & 0.8096 & 0.8872 & 0.7809 \\
\hline$\Delta_{2}(\boldsymbol{x})$ & 0.9760 & 1.0372 & 0.8880 & 0.9091 \\
\hline$\mu_{\Delta_{1}}\left(\Delta_{1}(\boldsymbol{x})\right)$ & & & & 0.9682 \\
\hline$\mu_{\Delta_{2}}\left(\Delta_{2}(\boldsymbol{x})\right)$ & & & 0.9829 & 0.9094 \\
\hline
\end{tabular}

Since DM1 is not satisfied with this solution, DM1 sets the minimal satisfactory level $\hat{\delta}_{1}$ to 0.80 . (12) For $\hat{\delta}_{1}=0.80(12)$ is solved, and the obtained optimal solution is $\mu_{1}\left(Z_{1}^{F}(\boldsymbol{x})\right)=0.8247, \mu_{2}\left(Z_{2}^{F}(\boldsymbol{x})\right)=0.6677$, and $\mu_{3}\left(Z_{3}^{F}(\boldsymbol{x})\right)=0.6925$, as is shown at the column labeled "2nd" in Table 1.

In step 5, DM2 sets the membership function $\mu_{\Delta_{2}}\left(\Delta_{2}(\boldsymbol{x})\right)$ for the ratio $\Delta_{2}$ of satisfactory degrees and the minimal satisfactory level as $\hat{\delta}_{\Delta_{2}}=0.80$. For $\hat{\delta}_{\Delta_{2}}=0.80$ (13) is solved, and the obtained result is shown at the column labeled "3rd" in Table 1. For the obtained optimal solution to $(13), \mu_{1}\left(Z_{1}^{F}(\boldsymbol{x})\right)=$ $0.8010, \mu_{2}\left(Z_{2}^{F}(\boldsymbol{x})\right)=0.7107, \mu_{3}\left(Z_{3}^{F}(\boldsymbol{x})\right)=0.6311$ and $\mu_{\Delta_{2}}\left(\Delta_{2}(\boldsymbol{x})\right)=0.9829$.

In step 6 , since the ratio of satisfactory degrees $\Delta_{2}$ is greater than $\hat{\delta}_{\Delta_{2}}=0.80$, the condition of termination of the interactive process is fulfilled. Then, DM1 is asked whether he is satisfied with the obtained solution. Since DM1 is not satisfied, and he updates the minimal satisfactory level $\hat{\delta}_{1}$ from 0.80 to 0.85 in order to improve $\mu_{1}\left(Z_{1}^{F}(\boldsymbol{x})\right)$ and sets $\hat{\delta}_{\Delta_{1}}=0.80$.

In step $7,(14)$ for $\hat{\delta}_{1}=0.85$ and $\hat{\delta}_{\Delta_{1}}=0.800$ is solved. The obtained result is shown at the column labeled " 4 th" in Table 1. For the obtained optimal solution to $(14), \mu_{1}\left(Z_{1}^{F}(\boldsymbol{x})\right)=0.8680, \mu_{2}\left(Z_{2}^{F}(\boldsymbol{x})\right)=0.6778$, $\mu_{3}\left(Z_{3}^{F}(\boldsymbol{x})\right)=0.6162$ and $\mu_{\Delta_{1}}\left(\Delta_{1}(\boldsymbol{x})\right)=0.9682$.

In step 6 , since the current solution satisfies all termination conditions of the interactive process and DM1 is satisfied with the current solution, the satisfactory solution is obtained and the interaction procedure is terminated.

\section{Conclusion}

In this paper, with simultaneously considering uncertainty and discreteness often appeared in the real world hierarchical decision making situations, we first formulated multi-level 0-1 programming problems with random variable coefficients in both objective functions and constraints. Through the use of fractile models together with chance constrained programming, the formulated stochastic multi-level 0-1 programming problems were transformed into deterministic 0-1 programming ones. Considering the vague nature of the DMs' judgments in the transformed multi-level 0-1 programming problems, interactive fuzzy programming has been proposed. In the proposed interactive method, after determining the fuzzy goals of the DMs at all levels, a satisfactory solution is derived efficiently by updating the satisfactory degree of the DM at the 1st level with considerations of overall satisfactory balance among all levels. It is significant to note here that the transformed deterministic problems to derive an overall satisfactory solution can be effectively solved through the proposed tabu search for general 0-1 programming problems. An illustrative numerical example for a three-level 0-1 programming problem was provided to demonstrate the feasibility of the proposed method. However, further computational experiences should be carried out for several types of numerical examples. From such experiences the proposed method must be revised. As a subject of future work, applications of the proposed method to the real world decision making situations should be considered in the near future. Extensions to other stochastic programming models will be considered elsewhere. Also extensions to multi-level 0-1 programming problems involving fuzzy random variable coefficients and/or random fuzzy coefficients will be required in the near future.

\section{REFERENCES}

[1] M. Sakawa, I. Nishizaki. Cooperative and Noncooperative Multi-Level Programming, Springer, New York, 2009.

[2] Y. J. Lai. Hierarchical optimization: a satisfactory solution, Fuzzy Sets and Systems, Vol. 77, No. 3, 321-335, 1996.

[3] H. S. Shih, Y. J. Lai, E. S. Lee. Fuzzy approach for multi-level programming problems, Computers and Operations Research, Vol. 23, No. 1, 73-91, 1996.

[4] M. Sakawa, I. Nishizaki, Y. Uemura. Interactive fuzzy programming for multi-level linear programming problems, Computers \& Mathematics with Applications, Vol. 36, No. 2, 71-86, 1998.

[5] M. Sakawa, I. Nishizaki, Y. Uemura. Interactive fuzzy programming for two-level linear fractional programming problems with fuzzy parameters, Fuzzy Sets and Systems, Vol. 115, No. 1, 93-103, 2000.

[6] M. Sakawa, I. Nishizaki. Interactive fuzzy programming for decentralized two-level linear programming problems, Fuzzy Sets and Systems, Vo. 125, No. 3, 301-315, 2002 .

[7] M. Sakawa, I. Nishizaki, Y. Uemura. A decentralized two-level transportation problem in a housing material manufacturer -Interactive fuzzy programming approach-, European Journal of Operational Research, Vol. 141, No. 1, 167-185, 2002.

[8] M. Sakawa. Fuzzy multiobjective and multilevel optimization, In M. Ehrgott, X. Gandibleux (Eds.), Multiple Criteria Optimization -State of the art annotated bibliographic surveys-, Kluwer Academic Publishers, Boston, 171-226, 2002.

[9] M. Sakawa, H. Katagiri. Interactive fuzzy programming based on fractile criterion optimization model for twolevel stochastic linear programming problems, Cybernetics and Systems, Vol. 41, 508-521, 2010. 
[10] M. Sakawa, H. Katagiri, T. Matsui. Interactive fuzzy random two-level linear programming through fractile criterion optimization, Mathematical and Computer Modelling, Vol. 54, 3153-3163, 2011.

[11] M. Sakawa, H. Katagiri, T. Matsui. Interactive fuzzy stochastic two-level integer programming through fractile criterion optimization, Operational Research: An International Journal, Vol. 12, pp. 209-227, 2012.

[12] M. Sakawa, T. Matsui. Interactive fuzzy programming for stochastic two-level linear programming problems through probability maximization, Artificial Intelligence Research, Vol. 2, 109-124, 2013.

[13] M. Sakawa, I. Nishizaki. Interactive fuzzy programming for multi-level programming problems: a review, International Journal of Multicriteria Decision Making, Vol. 2, No. 3, 241-266, 2012.

[14] I. M. Stancu-Minasian. Stochastic Programming with Multiple Objective Functions, D. Reidel Publishing Company, Dordrecht, 1984.

[15] I. M. Stancu-Minasian. Overview of different approaches for solving stochastic programming problems with multiple objective functions, In R. Slowinski, J. Teghem (Eds.), Stochastic Versus Fuzzy Approaches to Multiobjective Mathematical Programming under Uncertainty, Kulwer Academic Publishers, Dordrecht/Boston/London, 71-101, 1990.

[16] M. Sakawa, I. Nishizaki, H. Katagiri. Fuzzy Stochastic Multiobjective Programming, Springer, New York, 2011.

[17] H.-J. Zimmermann. Fuzzy programming and linear programming with several objective functions, Fuzzy Sets and Systems, Vol. 1, No. 1, 45-55, 1978.

[18] M. Sakawa. Fuzzy Sets and Interactive Multiobjective Optimization, Plenum Press, New York, 1993.

[19] M. Sakawa. Genetic Algorithms and Fuzzy Multiobjective Optimization, Kluwer Academic Publishers, Boston, 2001.

[20] G. B. Dantzig. Linear programming under uncertainty, Management Science, Vol. 1, No. 3-4, 197-206, 1955.

[21] A. Charnes, W. W. Cooper. Chance constrained programming, Management Science, Vol. 6, No. 1, 73-79, 1959.

[22] M. Sakawa, H. Yano, T. Yumine. An interactive fuzzy satisficing method for multiobjective linearprogramming problems and its application, IEEE Transactions on Systems, Man and Cybernetics, Vol. SMC-17, No. 4, 654-661, 1987.

[23] M. Sakawa, Fuzzy multiobjective optimization, In M. Doumpos, E. Grigoroudis (Eds.), Multicriteria Decision Aid and Artificial Intelligence: Links, Theory, and
Applications, John Wiley \& Sons, New York, 235-271, 2013.

[24] M. Sakawa, K. Kato. An interactive fuzzy satisficing method for multiobjective stochastic linear programming problems using chance constrained conditions, Journal of Multi-Criteria Decision Analysis, Vol. 11, No. 3, 125-137, 2002.

[25] M. Sakawa, K. Kato, I. Nishizaki. An interactive fuzzy satisficing method for multiobjective stochastic linear programming problems through an expectation model, European Journal of Operational Research, Vol. 145, No. 3, 655-672, 2003.

[26] M. Sakawa, K. Kato, H. Katagiri. An interactive fuzzy satisficing method for multiobjective linear programming problems with random variable coefficients through a probability maximization model, Fuzzy Sets and System, Vol. 146, No. 2, 205-220, 2004.

[27] M. Sakawa, H. Yano. An interactive fuzzy satisficing method using augmented minimax problems and its application to environmental systems, IEEE Transactions on Systems, Man and Cybernetics, Vol. SMC-15, No. 6, 720-729, 1985.

[28] M. Sakawa, H. Yano. Interactive decision making for multiobjective nonlinear programming problems with fuzzy parameters, Fuzzy Sets and Systems, Vol. 29, No. 3, 315-326, 1989.

[29] M. Sakawa, H. Yano. An interactive fuzzy satisficing method for generalized multiobjective linear programming problems with fuzzy parameters, Fuzzy Sets and Systems, Vol. 35, No. 2, 125-142, 1990.

[30] M. Sakawa, K. Kato. Interactive fuzzy multi-objective stochastic linear programming, In C. Kahraman (Ed.), Fuzzy Multi-Criteria Decision Making -Theory and Applications with Recent Developments-, Springer, New York, 375-408, 2008.

[31] A. Charnes, W. W. Cooper. Deterministic equivalents for optimizing and satisficing under chance constraints, Operations Research, Vol. 11, No. 1, 18-39, 1963.

[32] S. Kataoka. A stochastic programming model, Econometorica, Vol. 31, No. 1-2, 181-196, 1963.

[33] A. M. Geoffrion. Stochastic programming with aspiration or fractile criteria, Management Science, Vol. 13, No. 9, 672-679, 1967.

[34] S. Hanafi, A. Freville. An efficient tabu search approach for the 0-1 multidimensional knapsack problem, European Journal of Operational Research, Vol. 106, No. 2-3, 659-675, 1998 .

[35] R. E. Bellman, L. A. Zadeh. Decision making in a fuzzy environment, Management Science, Vol. 17, No. 4, 141$164,1970$. 